\title{
Extraction of knowledge on spatial distribution and spatial relationship from scanned topographic map using Convolutional Neural Networks
}

\author{
$\mathrm{Wu} \mathrm{Hao}{ }^{\text {a }}$, Hongguo Jia ${ }^{\text {a, }}$ * \\ ${ }^{a}$ Faculty of Geosciences and Environmental Engineering, Southwest Jiaotong University, Chengdu, Sichuan, China \\ WuHao,hwu@my.swjtu.edu.cn, HongguoJia, lemongg@home.swjtu.edu.cn \\ * Corresponding author
}

Keywords: Scanning topographic maps, Convolutional Neural Networks, Geographic knowledge

\begin{abstract}
:
Topographic maps (TM) contain plenty of geographic information, such as topographic fluctuations, hydrological networks, vegetation, administrative regions, residential areas, transportation routes and facilities and other man-made features. Based on geographic information, the map knowledge extracted from topographic maps has been widely used in many research fields, such as landscape ecology, land and resources management and urbanization.

Traditional topographic maps are generally in paper-format. It is difficult to use them for the spatial or multi-temporal analysis. Thus many research work focus on the extraction of geographic information based on scanned topographic maps (STM).Most of the existing studies developed many methods and algorithms to extract the geographical information from scanned topographic maps. However, these proposed methods usually only can extract a certain kind of feature, and parameters used in these methods are needed to set manually. However, for map knowledge, e.g. spatial distribution and spatial relationship among different map features, it is difficult to effectively combine different methods to extract map knowledge. Therefore, this paper proposes a method of extracting geographic knowledge based on deep-learning, which can be object-oriented and efficiently extract geographic knowledge. This method contains three steps: 1) establishing samples for different map features; 2) using the Convolutional Neural Networks (CNN), which is suited to the image recognition (Karpathy A et al. 2014), to classify the scanned topographic map; 3) estimating the proportion of different map features on maps and describing the spatial distribution based on a grid.
\end{abstract}

The method proposed in this study has been evaluated by some scale topographic maps. The results indicate that the extraction precise of this method can reach more than $70 \%$ for water and mountain areas and can also describe the spatial distribution for the features with larger map areas. 